Reprod. Nutr. Dévelop. 1980, 20 (4 A), 949-959.

\title{
Evolution comparée de la composition corporelle de lapins de trois types génétiques, au cours du développement postnatal
}

\author{
par J. OUHAYOUN \\ avec la collaboration technique de Danielle DELMAS \\ Laboratoire de Recherches sur l'Elevage du Lapin, I.N.R.A., \\ 31320 Castanet Tolosan, France.
}

Summary. Comparative development of the body components of three genetic types of rabbits during posinatal growth.

The postnatal growth of 45 male rabbits, from matings of Little Russian (PR), New Zealand White (NZ) or « Géant blanc du Bouscat 》 (GB) sire (widely different in mature body weight) with New Zealand White does has been studied between 14 and 84 days. Until 70 days, a strong maternal effect dissimulated the genetic potentialities of ponderal growth in the three types of rabbits (table 1). Those which had the highest mature body weight due to their genetic origin, showed the fastest ponderal growth rate but were the last to attain a given degree of maturity in body weight. The development of carcass components was studied using allometric relations (table 3 ). The classification of body components in decreasing order of precocity (increasing o der of allometric coefficients) was as follows : bone tissue, muscle tissue, DNA, minerals, water, proteins and lipids for biochemical components. The allometric coefficients of bone and muscle tissues, water, lipids and DNA differed in the three genetic types, explaining the differences in body composition observed among those types compared at equal age, weight or degree of maturity in body weight (table 4). The body composition depended not only on the physiological stage, but also on the rate at which it was attained. The progeny of « Géant blanc du Bouscat » had low carcass qualities : reduced adiposity and muscle/bone ratio ; Little Russians and New Zealands showed higher precocity : at all the stages studied, the $(P R \times N Z)$ rabbits exhibited the highest carcass adiposity, while the New Zealand rabbits had the best muscle/bone ratio.

\section{Introduction.}

Depuis les études de Cantier et al. (1969.1974), Baron et Vigneron (1970), Vezinhet ef al., (1972) et celles de Dulor et al. (1976), les modalités de la croissance relative des principaux organes et tissus du Lapin commun sont bien connues. Sur le plan zootechnique, les résultats acquis permettent d'interpréter les variations des caractéristiques bouchères du Lapin, au cours du développement, et de définir les âges optimaux d'abattage, compte-tenu, d'une part, des contraintes liées à l'amélioration de la productivité de l'élevage et, d'autre part, de la nécessité de maintenir une production 
de qualité. A cet égard, les caractéristiques bouchères les plus importantes sont le rendement en carcasse, le rapport muscle/os et l'adiposité de la carcasse. Rouvier (1970) a mis en évidence une variabilité génétique entre souches de ces caractères. Cela implique l'existence de relations propres à chaque génotype entre la taille, la forme corporelle et l'âge.

Dans la présente étude du développement du Lapin, nous nous proposons d'analyser la mise en place des différences de composition corporelle entre types génétiques. Pour cela, nous nous intéressons, non seulement à l'expansion pondérale du corps en fonction du temps, mais aussi à ses changements de forme, c'est-à-dire aux modifications des proportions des constituants tissulaires, cellulaires et chimiques en fonction de la taille.

\section{Matériel et méthodes.}

1. Matériel animal. - L'étude porte sur 45 lapins mâles échantiltonnés parmi les produits de la troisième portée de femelles néozélandaises (NZ) accouplées à des mâles, soił géants blancs du Bouscat (GB) ( 3 pères, 5 mères), soit néozélandais ( 3 pères, 7 mères), soit petits russes (PR) (4 pères, 6 mères). Deux types de métis sont donc comparés à une souche pure. Les lapereaux sont sexés ef indentifiés à l'âge de 10 jours. Les différences entre les effectifs moyens des portées allaitées (GB $\times N Z: 8,80 \pm 0,73$; $N Z \times N Z: 7,57 \pm 1,13 ; P R \times N Z: 8,00 \pm 0,93)$ ne sont pas significatives à 14 jours. Au sevrage (28 jours), les mâles sont placés en cages individuelles grillagées et nourris à volonté avec un aliment granulé complet du commerce, dosant 14 p. 100 minimum de matières azotées totales et 15 p. 100 maximum de cellulose. A cinq stades du développement : 14 jours, 28, 42, 70 ou 84 jours, trois lapins sont tirés au hasard dans chacune des trois populations et sacrifiés.

2. Caractères mesurés et méthodes analytiques. - Les lapereaux sont caractérisés par leur poids vif à chacun des stades d'étude et par leur vitesse de croissance exprimée en gain de poids corporel entre ces stades. Les sujets qui sont échantillonnés pour analyse ne sont pas soumis au jeûne, préalablement au sacrifice.

Dès l'abattage, le corps est diminué de la peau, des viscères abdominaux ef thoraciques, du cerveau et de la moelle épinière. L'ensemble obtenu, composé pour l'essentiel des tissus osseux, musculaire et adipeux est divisé sagittalement. Sur une demi-《 carcasse », le membre postérieur est prélevé en respectant des repères anatomiques précis (Vezinhet el al., 1972), puis désossé. Les tissus constitutifs sont pesés. Le rapport muscle/os, qui en est déduit, esł représentatif de celui de la carcasse, à un facteur constant près. En effet, l'isométrie de développement de la musculature totale et des muscles du membre postérieur, d'une part, du squelette total et des os de ce groupe anatomique, d'autre part, a été montrée par Vezinhet et al. (1972) et Culor et al. (1976), respectivement. L'autre demi-carcasse est pesée puis immédiatement immergée dans l'azote liquide. Elle est finement broyée dans un hachoir Scharfen puis dans un homogenéiseur Turmix. Les analyses chimiques portent sur l'homogénat lyophilisé. L'eau est estimée par chauffage à $103^{\circ} \mathrm{C}$, pendant $24 \mathrm{~h}$, d'une prise d'essai de $5 \mathrm{~g}$ environ ; les sels minéraux sont obtenus par calcination à $530^{\circ} \mathrm{C}$ pendant $6 \mathrm{~h}$ de la matière sèche obtenue. L'azote est dosé par minéralisation 
de $0,2 \mathrm{~g}$ de lyophilisat par la méthode de Kjeldahl puis par mesure colorimétrique de l'ammoniac (chaîne Technicon) ; les lipides sont extraits selon la technique de Pinlokaya (1958). L'ADN est dosé selon une technique voisine de celle utilisée par Durand ef al. (1965). L'ADN est séparé de l'ARN par la méthode de Schmidt et Thannhauser (1945). Les bases puriques, provenant de son hydrolyse perchlorique ménagée, sont dosées par spectrophotométrie UV après séparation sur résine échangeuse de cations (amberlite CG 120). Dans la mesure où la quantité d'ADN contenue dans un noyau diploïde est constante (Boivin, Vendrely et Vendrely, 1948), les variations du nombre et de la taille moyenne des cellules peuvent être déduites, respectivement, des variations de la quantité totale d'ADN et du rapport : poids du tissu/ADN total. La « cellule », selon le concept de Enesco et Leblond (1962) représente, dans l'ensemble de tissus considéré, un noyau ef la quantité moyenne de matière vivante qui lui est associée. Elle est donc virtuelle.

3. Méthodes statistiques. - Les paramètres statistiques des séries de mesures (moyennes, écarts-types) sont calculés par type génétique, en fonction d'un facteur contrôlé (temps). L'effet du type génétique sur les moyennes est estimé par analyse de variance à un facteur de classification.

L'évolution relative des caractères anatomiques ef biochimiques, au cours du développement, est étudiée par la relation d'allométrie : $y=b^{a}$, à l'instar de Cantier ef al. (1969). Le coefficient a d'allométrie représente le rapport des vitesses spécifiques de croissance de deux parties $y$ et $x$ de l'organisme :

$$
\frac{d y}{y d t} / \frac{d x}{x d t} \text {. }
$$

Les variables de référence utilisées sont le poids vif et la demi-carcasse définie ci-dessus, constituée des tissus osseux, musculaire et adipeux. Le calcul des coefficients de la relation d'allométrie est effectué par la méthode des moindres carrés. Les coefficients a sont les pentes des droites représentatives des équations de régression de $\log _{\mathrm{e}} \mathrm{y}$ sur $\log _{e} x$ afférentes à l'ensemble de la période analysée $: \log _{e} y=a \log _{e} x+\log b$. Du fait de la faible taille des échantillons d'animaux, d'éventuelles discontinuités dans la croissance relative, s'exprimant par des variations des coefficients d'allométrie, n'ont pas été recherchées. Les coefficients d'allométrie homologues, calculés pour chacun des trois types génétiques, sont comparés selon la méthode de Dagnelie (1970).

Enfin, une analyse de covariance à un facteur contrôlé (génotype) est effectuée, en considérant le poids de carcasse comme covariable. Les variables introduites dans cette analyse sont le poids vif, les composantes tissulaires ef chimiques de la carcasse.

\section{Résultats et discussion.}

Le développement pondéral ef l'évolution de la composition corporelle des jeunes dépendent de leur propre génotype, qui résulte de l'égale contribution des parents (effets génétiques directs ef d'interaction), des effets du milieu et de l'influence maternelle. Compte-tenu du schéma expérimental et des effectifs d'animaux mis en œuvre, l'analyse de ces facteurs de variation n'est pas envisagée dans la présente 


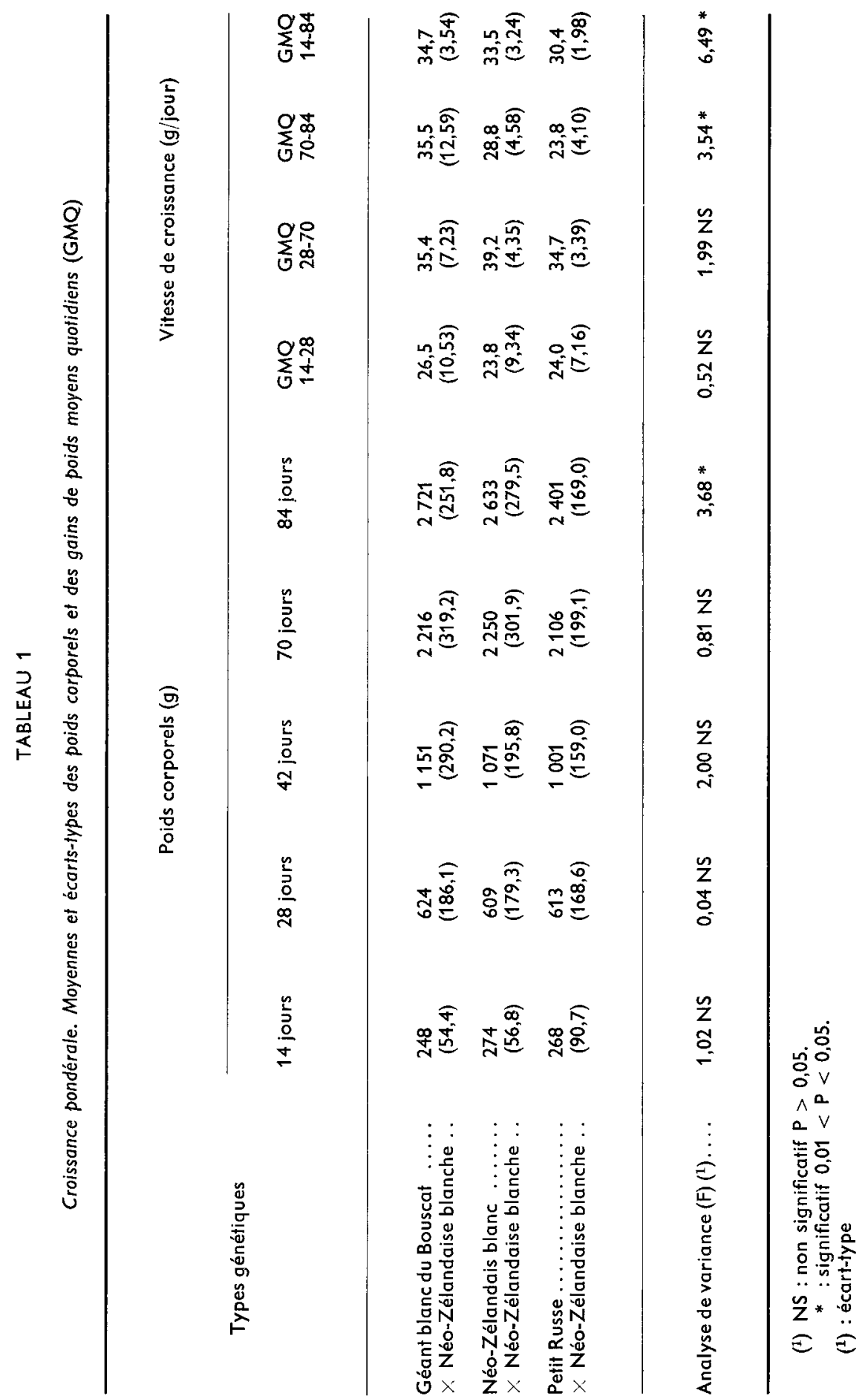


étude. Celle-ci ne les aborde que dans la globalité de leur expression chez les trois types génétiques comparés, qui ont la même origine maternelle mais des origines paternelles distinctes.

1. Croissance pondérale. - Alors que les écarts entre les poids adultes des souches paternelles sont très grands (Géants blancs du Bouscat: $5,5 \mathrm{~kg}$; Néozélandais blancs : $4,1 \mathrm{~kg}$; Petits Russes : $2,5 \mathrm{~kg}$ ), les caractères de croissance pondérale de leurs descendants ne diffèrent pas de façon significative jusqu'à l'âge de 70 jours (tabl. 1). Cela esł dû à la forte rémanence de l'influence maternelle qui, initialement, favorise les produits des mâles de formats moyen et petit, mais est insuffisante pour permettre aux descendants des mâles géants d'extérioriser au mieux leur potentiel génétique de croissance (Venge, 1950 ; Ouhayoun, 1978). Au-delà de l'âge de 70 jours, l'effet différentiel de chaque souche paternelle se révèle : les poids adultes moyens des mâles et les vitesses moyennes de croissance de leurs descendants, entre 70 et 84 jours, sont en corrélation élevée $(r=0,99 * *)$. Toutefois, à l'âge de 84 jours, les différences entre les poids corporels moyens, bien que favorables aux croisés ( $G B \times N Z$ ) et aux sujets néozélandais, sont toujours faibles, eu égard aux écarts existant, à l'état adulte, entre les types génétiques comparés (voir tabl. 2).

TABLEAU 2

\begin{tabular}{|c|c|c|}
\hline & Poids adulte & Poids à 84 jours \\
\hline $\begin{array}{l}G B \times N Z \ldots \\
N Z \times N Z \ldots \\
P R \times N Z \ldots\end{array}$ & $\begin{array}{l}4,80 \mathrm{~kg}(145) \\
4,10 \mathrm{~kg}(124) \\
3,30 \mathrm{~kg}(100)\end{array}$ & $\begin{array}{l}2,72 \mathrm{~kg}(113) \\
2,63 \mathrm{~kg}(110) \\
2,40 \mathrm{~kg}(100)\end{array}$ \\
\hline
\end{tabular}

( ) : Valeurs calculées par référence aux croisés PR $\% \mathrm{NZ}$.

Globalement, la précocité de croissance des lapereaux, estimée par le degré de maturité pondérale atteint à l'âge de 84 jours (pourcentage du poids adulte) est plus grande chez les types génétiques des plus petits formats adultes ( $P R \times N Z: 72,8$ p. $100 ; N Z \times N Z: 64,2$ p. $100 ; G B \times N Z: 56,7$ p. 100).

2. Développement de la carcasse. - Le développement de la carcasse, par rapport au corps entier, suit une loi d'allométrie majorante entre 14 et 84 jours. Ce résultat est complémentaire de ceux de Cantier et al. (1969). En effet, ces auteurs ont montré, chez leur souche expérimentale, étudiée entre 9 ef 182 jours, que les organes suivants : cerveau, trachée-cœur-poumons, reins, tractus digestif, peau et foie, qui sont prélevés au cours de la préparation de la carcasse, telle qu'elle est définie dans la présente étude, suivent, à l'inverse, une loi d'allométrie minorante, par rapport au « poids vif vide ». Les coefficients d'allométrie $\left(a \pm s_{a}\right)$ des trois types génétiques ne diffèrent pas significativement : $\mathrm{GB} \times \mathrm{NZ}: 1,07 \pm 0,03 ; \mathrm{NZ} \times \mathrm{NZ}: 1,11 \pm 0,02 ; \mathrm{PR} \times \mathrm{NZ}:$ $1,09 \pm 0,02$.

La fraction du poids corporel représentée par la carcasse, passe de 42 p. 100 à 14 jours à 55 p. 100 à 84 jours, soit entre les poids de 250-275 g et $2400-2720 \mathrm{~g}$, selon 
les types génétiques (fig. 1). Les croisés $(G B \times N Z$ ) ont, en moyenne, le plus faible développement relatif de la carcasse, surtouł jusqu'à l'âge de 70 jours; mais les écarts avec les deux autres types génétiques ne sont pas significatifs.

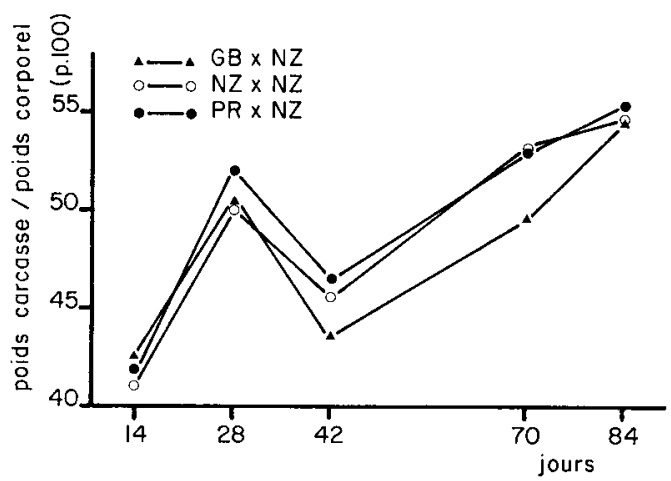

FIG. 1. - Evolution, en fonction de l'âge, de la fraction du poids corporel représentée par la carcasse.

TABLEAU 3

Relation du logarithme du poids de chaque constifuant de la carcasse (y) ef du logarithme du poids de la carcasse $(x)$ (les bornes de $y$ et de $x$ sont en données naturelles)

\begin{tabular}{|c|c|c|c|c|c|c|c|}
\hline & Variables & & Paramètres & $\mathrm{GB} \times \mathrm{NZ}$ & $N Z \times N Z$ & $\mathrm{PR} \times N Z$ & $F\left({ }^{1}\right)$ \\
\hline \multirow{7}{*}{$\begin{array}{l}\text { y (variables } \\
\text { dépendantes) }\end{array}$} & \multirow{2}{*}{$\begin{array}{c}\text { Tissus } \\
\text { du membre } \\
\text { postérieur }\end{array}$} & $\begin{array}{l}\text { Os } \\
(g)\end{array}$ & $\begin{array}{c}a \pm s_{a} \\
\text { bornes de y }\end{array}$ & $\begin{array}{c}0,83 \pm 0,03 \\
2,8-34,1\end{array}$ & $\begin{array}{c}0,77 \pm 0,03 \\
2,7-23,8\end{array}$ & $\begin{array}{l}0,76 \pm 0,03 \\
2,0-24,6\end{array}$ & $\begin{array}{l}1,90 \\
\text { NS }\end{array}$ \\
\hline & & $\begin{array}{l}\text { Muscle } \\
\text { (g) }\end{array}$ & $\begin{array}{c}a \pm s_{a} \\
\text { bornes de y }\end{array}$ & $\begin{array}{c}1,15 \pm 0,01 \\
5,9-218,0\end{array}$ & $\begin{array}{c}1,11 \pm 0,02 \\
7,1-173,6\end{array}$ & $\begin{array}{c}1,11 \pm 0,01 \\
4,7-171,6\end{array}$ & $\begin{array}{c}2,57 \\
\text { NS }\end{array}$ \\
\hline & \multirow{5}{*}{$\begin{array}{l}\text { Composants } \\
\text { de la demi- } \\
\text { carcasse }\end{array}$} & $\begin{array}{l}\text { ADN } \\
(\mathrm{mg})\end{array}$ & $\begin{array}{c}a \pm s_{a} \\
\text { bornes de y }\end{array}$ & $\begin{array}{l}0,67 \pm 0,03 \\
339-2723\end{array}$ & $\begin{array}{c}0,62 \pm 0,03 \\
373-2187\end{array}$ & $\begin{array}{l}0,61 \pm 0,02 \\
271-2125\end{array}$ & $\begin{array}{r}2,16 \\
\text { NS }\end{array}$ \\
\hline & & $\begin{array}{c}\text { Minéraux } \\
\text { (g) }\end{array}$ & $\begin{array}{c}a \pm \mathbf{s}_{\mathbf{a}} \\
\text { bornes de } y\end{array}$ & $\begin{array}{c}0,95 \pm 0,03 \\
2,0-38,9\end{array}$ & $\begin{array}{c}0,90 \pm 0,02 \\
2,9-38,6\end{array}$ & $\begin{array}{c}0,90 \pm 0,02 \\
1,8-36,2\end{array}$ & $\begin{array}{l}1,68 \\
\text { NS } \\
\end{array}$ \\
\hline & & $\begin{array}{c}\text { Eau } \\
(g)\end{array}$ & $\begin{array}{c}a \pm s_{\mathrm{a}} \\
\text { bornes de } y\end{array}$ & $\begin{array}{c}0,97 \pm 0,01 \\
28,4-586,3\end{array}$ & $\begin{array}{c}0,96 \pm 0,01 \\
31,0-466,3\end{array}$ & $\begin{array}{c}0,94 \pm 0,01 \\
21,4-435,9\end{array}$ & $\begin{array}{c}3,90 \\
*\end{array}$ \\
\hline & & $\begin{array}{l}\text { Protéines } \\
\text { (g) }\end{array}$ & $\begin{array}{c}a \pm s_{a} \\
\text { bornes de } y\end{array}$ & $\begin{array}{c}1,05 \pm 0,01 \\
6,7-174,6\end{array}$ & $\begin{array}{c}1,06 \pm 0,01 \\
7,2-136,1\end{array}$ & $\begin{array}{c}1,05 \pm 0,02 \\
4,7-125,5\end{array}$ & $\begin{array}{c}0,13 \\
\text { NS }\end{array}$ \\
\hline & & $\begin{array}{l}\text { Lipides } \\
(g)\end{array}$ & $\begin{array}{c}a \pm s_{a} \\
\text { bornes dey }\end{array}$ & $\begin{array}{c}1,14 \pm 0,05 \\
1,7-80,2\end{array}$ & $\begin{array}{c}1,22 \pm 0,08 \\
2,5-93,0\end{array}$ & $\begin{array}{c}1,37 \pm 0,08 \\
1,3-131,5\end{array}$ & $\begin{array}{l}3,33 \\
\text { NS }\left(^{2}\right)\end{array}$ \\
\hline \multicolumn{3}{|c|}{$\begin{array}{c}x \text { (variable indépendante) } \\
1 / 2 \text { carcasse }(g)\end{array}$} & bornes de $x$ & $38,8-880,0$ & $43,6-729,5$ & $29,2-729,1$ & \\
\hline
\end{tabular}

(1) NS : non significatif $(P>0,05)$.

* : significatif $(0,01<P<0,05)$.

(2) $0,05<P<0,1$. 
L'augmentation du rapport du poids de la carcasse au poids corporel subit un accident apparent entre 14 et 42 jours, commun aux trois types de lapereaux : l'accroissement rapide entre 14 et 28 jours est suivi d'un sensible infléchissement postsevrage. Cette discontinuité est probablement due, à la fois, aux changements d'allométrie du tube digestif et de la peau, aux «poids vifs vides» de $550-730 \mathrm{~g}$ et de $790-890 \mathrm{~g}$, respectivement (Cantier ef al., 1969), et à la variation relative du contenu digestif consécutive au changement de régime et de comportement alimentaire au sevrage (28 jours).

3. Développement relatif des composants de la carcasse. - Les coefficients d'allométrie des tissus du membre postérieur et des constituants chimiques de la carcasse sont présentés dans le tableau 3.

La croissance de la musculature du membre postérieur, par rapport à la croissance de la carcasse, suit une loi d'allométrie majorante ; au contraire, l'allométrie des os est minorante. Il en résulte un accroissement du rapport muscle/os au cours du développement (fig. 2).

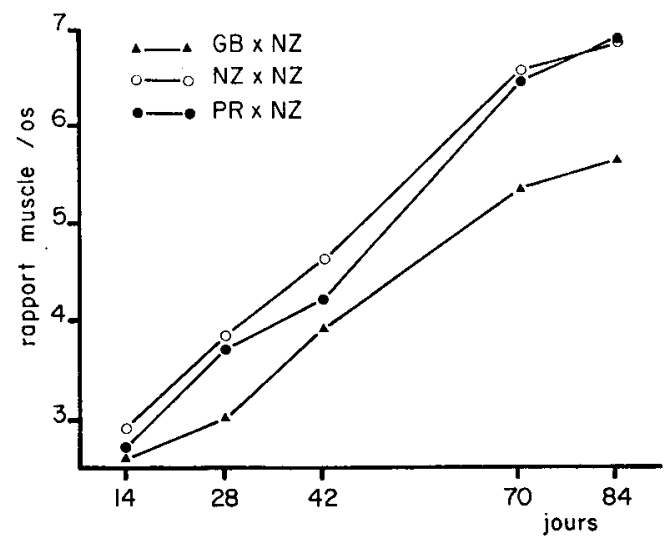

FIG. 2. - Evolution, en fonction de l'âge, du rapport muscle/os du membre postérieur.

Les constituants chimiques de la carcasse se répartissent en deux groupes : l'eau, les minéraux et l'acide désoxyribonucléique manifestent une allométrie minorante alors que les protéines, mais surtout les lipides, se caractérisent par une allométrie majorante. En conséquence, les teneurs de la carcasse en ces trois premiers constituants diminuent pendant la période étudiée, alors que les taux de lipides ef de protéines augmentent.

L'expansion pondérale de la carcasse est donc accompagnée de modifications qualitatives, tant au plan anatomique, qu'aux niveaux cellulaire et biochimique. La gradation dans la vitesse de croissance relative des composants chimiques mesurés, qui est commune aux trois types génétiques, est la suivante : acide désoxyribonucléique $(A D N)$, éléments minéraux, eau, protéines, lipides. L'ADN présente le coefficient d'allométrie le plus faible, ce qui traduit sa précocité de synthèse. Celle-ci est, en effet, active surtout au cours des premières étapes du développement, c'est-à-dire, au moment où la contribution de l'hyperplasie cellulaire à l'accroissement pondéral de 
l'organisme est prépondérante. Puis, l'hypertrophie des cellules constiłutives se manifeste par une réduction de la concentration en ADN ; l'accroissement de la masse moyenne des cellules, selon le concept de Enesco et Leblond (1962), est globalement, suivi par la dilution du matériel nucléaire. Dans la présente étude, le facteur de dilution de l'ADN dans la carcasse, entre 14 et 84 jours est faible, de l'ordre de 2,2-2,8 ; ce résultat s'accorde avec les observations de Cordesse et Nougues (1973) et Ouhayoun et Rouvier (1973), selon lesquelles le développement du tissu musculaire, qui représente de 75 à 85 p. 100 (entre 14 et 84 jours) du poids de la carcasse, procède plus d'une hyperplasie que d'une hypertrophie des cellules. La valeur des coefficients d'allométrie des éléments minéraux de la carcasse, minorante mais supérieure à celle des coefficients d'allométrie du tissu osseux, rend compte de la calcification de l'osséine, qui est une phase relativement peu précoce de la croissance du squelette. La réduction de la teneur en eau de la carcasse observée entre 2 et 12 semaines est accompagnée d'une augmentation corrélative de la teneur en lipides. La complémentarité de ces deux constituants chimiques corporels a été analysée par Vigneron et al. (1971), qui distinguent, en fait, deux périodes successives limitées par le poids vif de $1500-1780 \mathrm{~g}$ chez les lapereaux mâles de leur souche expérimentale. En deça de ce seuil, l'eau ef les lipides ont une croissance isométrique à celle du corps ; au-delà, l'allométrie de l'eau est minorante alors que celle des lipides est fortement majorante.

Les mécanismes de développement des constituants corporels analysés, anatomiques ou biochimiques, sont communs aux trois types génétiques comparés. Ni l'ordre de précocité, pas plus que le sens de la variation pondérale de chacun d'eux, par rapport à la carcasse, ne permettent de distinguer les groupes expérimentaux. Cependant, les valeurs des coefficients d'allométrie varient entre types génétiques. Bien que seuls les écarts entre les coefficients d'allométrie de l'eau $(0,01<P<0,05)$ et des lipides $(0,05<P<0,1)$ aient une signification statistique, deux tendances générales se dégagent. Tout d'abord, les lapins croisés de pères géants blancs du Bouscat se distinguent de l'ensemble des deux autres types génétiques; ensuite, les valeurs des coefficients d'allométrie varient avec le format adulte des lapins comparés ; soit dans le même sens (muscle ef os du membre postérieur, eau, ADN et éléments minéraux de la carcasse), soit en sens inverse (lipides de la carcasse). Il en résulte des états constitutifs propres à chaque type génétique. Les écarts observés dépendent du stade auquel est effectuée la comparaison : âge constant, poids de carcasse ou degré de maturité pondérale constants (tabl. 4).

Les carcasses des lapereaux $(G B \times N Z)$, qui se caractérisent, à la fois, par un faible degré de maturité pondérale aux âges (14 jours et 84 jours) ou au poids de carcasse $(1200 \mathrm{~g})$ envisagés, et par un long délai d'atteinte d'un degré de maturité pondérale donné (50 p. 100), sont moins riches en matière sèche et en lipides; elles présentent une concentration en ADN supérieure : les cellules virtuelles constitutives des carcasses sont donc, en moyenne, plus petites. II est possible que celles-ci aient des potentialités de croissance égales à celles des autres types génétiques, mais elles ne sont exprimées à aucun des stades choisis ; le rapport muscle/os est, en conséquence, particulièrement faible. Les carcasses des lapins néozélandais et $(P R \times N Z)$ présentent des similitudes, tant à âge égal (84 jours) qu'à poids de carcasse égal (1 $200 \mathrm{~g}$ ), c'est-àdire à degrés de maturité différents : les cellules virtuelles constitutives ont des masses moyennes très voisines, les rapports muscle/os moyens des membres postérieurs sont 


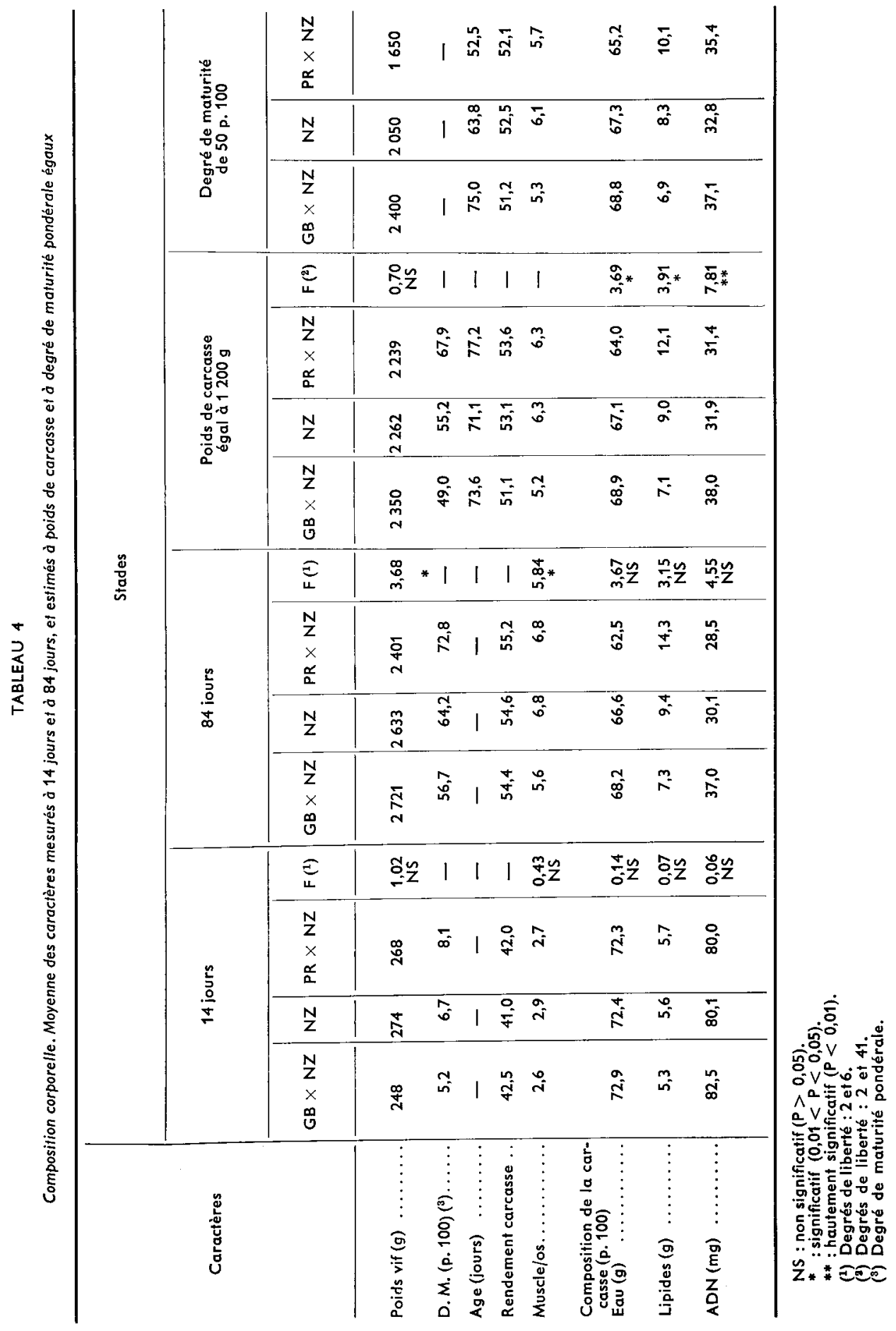


égaux. Cependant, les carcasses diffèrent par les teneurs en matière sèche et en lipides. Celles-ci sont fortes chez les croisés $(P R \times N Z)$, qui se caractérisent par les degrés de maturité les plus élevés. Il existe donc une variabilité entre souche de l'adiposité corporelle à rapport muscle/os constant. Enfin, la comparaison des trois lots expérimentaux au même degré de maturité pondérale fait apparaître des différences sensibles et ordonnées entre les teneurs en eau et en lipides des carcasses. Les types génétiques qui atteignent le plus vite le degré de maturité pondérale de $50 \mathrm{p} .100$ ont les carcasses les plus grasses et, corrélativement, les plus pauvres en eau. La vitesse d'atteinte d'un degré de maturité pondérale donné ne semble pas avoir d'influence sur l'expression des potentialités de croissance des cellules de la carcasse et, par conséquent sur le rapport muscle/os. Par ailleurs, bien qu'évoluant dans le même sens au cours de la vie postnatale, compte-tenu des modalités de la croissance relative des tissus et des organes, le rendement en carcasse ef le rapport muscle/os du membre postérieur se révèlent peu liés. Cela confirme des observations antérieures (Ouhayoun, 1978). Certes, un rendement en carcasse élevé est un critère de maturité générale de l'animal mais il ne représente pas une présomption de fort développement musculaire, relativement au squelette.

\section{Conclusion.}

L'utilisation de la relation d'allométrie dans l'étude comparée de l'évolution de la composition corporelle de lapins d'origines génétiques différentes, a permis de vérifier ou de montrer que les principaux composants corporels se développent selon des gradients de précocité. En ce qui concerne les tissus osseux et musculaire d'un groupe anatomique particulier : le membre postérieur, les résultats de Cantier ef al. (1969), obtenus sur une souche commune, sont vérifiés et étendus à d'autres types génétiques. Au plan des composants chimique de la carcasse, l'ordre de précocité suivant est observé : ADN, éléments minéraux, eau, protéines, lipides. Les trois premiers composants se caractérisent par une allométrie minorante dans la période étudiée ; les suivants, notamment les lipides, présentent une allométrie majorante. Le classement est commun aux trois types génétiques.

Une variabilité des coefficients d'allométrie esł mise en évidence, qui est à l'origine des différences de composition corporelle observées à âge égal ou estimées à poids de carcasse ou à degré de maturité constants. La composition corporelle varie, non seulement en fonction du stade physiologique, mais aussi en fonction de la vitesse avec laquelle celui-ci est atteint. Ce dernier facteur dépend des potentialités génétiques propres à chaque souche ou à chaque individu, mais aussi du milieu dans lequel elles sont appelées à s'extérioriser. A cet égard, chez de jeunes animaux, l'influence maternelle est essentielle. 


\section{Références}

BARON R., VIGNERON P., 1970. Hétérogonie biochimique des composants azotés du grand psoas au cours de la croissance postnatale du lapin. Ann. Biol. anim. Bioch. Biophys., 10, 223-238.

BOIVIN A., VENDRELY R., VENDRELY C., 1948. L'acide desoxyribonucléique du noyau cellulaire dépositaire des caractères héréditaires; arguments d'ordre analytique. C. R. Acad. Sci. Paris, 226, $1061-1062$.

CANTIER J., VEZINHET A., ROUVIER R., DAUZIER L., 1969. Allométrie de croissance chez le lapin (Oryctolagus cuniculus). I. Principaux organes et tissus. Ann. Biol. anim. Bioch. Biophys., 9, 5-39.

CANTIER J., VEZINHET A., DULOR J. P. ROUVIER R., 1974. Allométrie de croissance chez le lapin. IV. Principaux muscles de la carcasse. Ann. Biol. anim. Bioch. Biophys., 14, 271-292.

CORDESSE R. NOUGUES J., 1973. Multiplication et grandissement cellulaire, activité cellulaire du tissu musculaire du Lapin au cours de la croissance. I. Aspect descriptif. Ann. Biol. anim. Bioch. Biophys., 13, 535-552.

DAGNELIE P., 1970. Théorie et méthodes statistiques, Vol. 2, 281-287. Gembloux, Ed. Duculot.

DULOR J. P., VEZINHET A., CANTIER J., ROUVIER R., 1976. Allométrie de croissance chez le Lapin (Oryctolagus cuniculus). V. Le squelette. Ann. Biol. onim. Bioch. Biophys., 16, 569-577.

DURAND G., FAUCONNEAU G., PENOT E., 1965. Etude biochimique de la croissance de l'intestin grêle, du foie et de la carcasse du rat ; rôles respectifs de la multiplication et du grandissement cellulaires. Ann. Biol. Anim. Bioch. Biophys., 5, 163-187.

ENESCO M., LEBLOND C. P., 1962. Increase in cell number as a factor in the growth of the organs and tissues of the young male rat. J. Embryol. exp. Morph., 10, 530-532.

OUHAYOUN J., 1978. Elude comparative de races de lapins différant par le poids adulte. Incidence du format paternel sur les composantes de la croissance des lapereaux issus de croisement terminal. Th. $3^{\text {e }}$ cycle. Fac. Sci. Montpellier.

OUHAYOUN J., ROUVIER R., 1973. Rôle de la multiplication nucléaire ef du grandissement cellulaire dans la croissance musculaire de Lapins de plusieurs génotypes. Journ. Rech. avic. cunic., Paris, comm. IV. 3.

PINLOKAYA U., 1958. Myasnaya Ind. S.5.S.R., 29, 9. In Les Cahiers techniques du CNERNA. 8. Les méthodes analytiques des lipides simples. (PAQUOT C. et al.), p. 22. Publ. C.N.R.S., Paris.

SCHMIDT G., THANNHAUSER S. J., 1945. A method for the determination of desoxyribonucleic acid, ribonucleic acid and phosphoproteins in animal tissues. J. biol. Chem., 161, 83-89.

ROUVIER R., 1970. Variabilité génétique du rendement à l'abattage et de la composition anatomique de lapins de trois races. Ann. Génét. Sél. anim., 2, 325-346.

VENGE O., 1953. Studies on the maternal influence on the growth in rabbits. Acta. agric. Scand., 3, 243-291.

VEZINHET A., ROUVIER R., DULOR J. P., CANTIER J., 1972. Allométrie de croissance chez le lapin. III. Principales régions du système musculaire. Ann. Biol. anim. Bioch. Biophys., 12, 33-45.

VIGNERON P., BARON R., DAUZIER L., 1971. Evolution postnatale de la quantité d'eau et de lipides du corps ef du grand psoas chez le Lapin. Ann. Biol. anim. Bioch. Biophys., 11, 669-679. 